\title{
La adicción al juego. Referentes claves para los procesos de intervención psicossocial
}

\section{The game addiction. Key reference factors for psychosocial intervention processes}

DOI: $10.46932 / \mathrm{sfjdv2n5-071}$

Received in: Oct 1st, 2021

Accepted in: Dec 30th, 2021

\author{
Dra. Pilar Blanco Miguel \\ Doctora por la Universidad de Huelva. \\ Licenciada en Ciencias Políticas y Sociología. \\ Diplomada en Trabajo Social. Profesora adscrita al Departamento de Sociología, Trabajo Social y Salud \\ Pública de la Universidad de Huelva. España. \\ E-mail: pblanco@uhu.es \\ Dra. Yolanda Borrego Alés \\ Doctora por la Universidad de Huelva. \\ Licenciada en Psicología. \\ Profesora adscrita al Departamento de Psicología Social, Evolutiva y de la Educación de la Universidad \\ de Huelva. España.
}

\begin{abstract}
RESUMEN
Introducción. ¿Es posible dejar de jugar? Esta es una pregunta que cada día se hacen muchas personas cuando su situación de juego les pone entre la espada y la pared, haciéndoles pensar si existe una salida real a su problema. En la actualidad, las posibilidades de tratamiento y prevención del juego patológico son claras y patentes, aunque debemos de tener en cuenta tres cuestiones básicas: la necesidad de hacer una correcta identificación del problema, una consideración profesional del caso y su remisión a los dispositivos especializados para su tratamiento. Objetivos. Identificar las situaciones en las que un adicto o adicta al juego es capaz de plantearse dejar de jugar y por tanto iniciar el proceso de rehabilitación. Conocer qué implicaciones tiene esa decisión a nivel individual y familiar y que vienen a determinar el proceso de rehabilitación. Metodología. Se ha optado por una perspectiva metodológica que se sirva de estrategias que permitan conocer el fenómeno de la adicción al juego de mano de los propios afectados. De entre las técnicas posibles, se ha optado por la Historia de Vida y la Entrevista en Profundidad. Resultados. De manera general hemos avistado que, tarde o temprano, el ludópata se ve obligado tener que confesar la adicción a su familia, ya que su situación de juego se hace insostenible. De igual modo, hemos podido ver que, afrontar el problema debe ir más allá del loable propósito de encararlo de forma individual, ya que esto no suele funcionar, y que es necesario, tanto la ayuda familiar, como la profesional para poder llevar a cabo el proceso de rehabilitación con una cierta garantía de poder superar la adicción. Conclusiones. Es fácil advertir que para dejar de jugar tienen que operarse un gran cambio en el pensamiento del ludópata, es decir, tomarse en serio el querer dejar de jugar y partir de ahí solicitar la colaboración de los profesionales (entre ellos el Trabajador Social); ayuda que debe ser complementada con el apoyo familiar, quedando ambos determinados como los principales factores a tener en cuanta en los procesos de recuperación.
\end{abstract}

Palabras Claves: Adicción al juego, Afrontamiento del problema, Referentes Claves, Proceso de Rehabilitación, Intervención Psicosocial. 


\section{ABSTRACT}

Introduction. Is it possible to stop playing? This is a question that many people ask themselves every day when their gaming situation puts them between a rock and a hard place, making them wonder if there is a real way out of their problem. At present, the possibilities of treatment and prevention of pathological gambling are clear and patent, although we must take into account three basic questions: the need to make a correct identification of the problem, a professional consideration of the case and its referral to specialized devices for your treatment. Objectives. Identify the situations in which a gambling addict is able to consider stopping gambling and therefore start the rehabilitation process. Knowing what implications this decision has at the individual and family level and what come to determine the rehabilitation process. Methodology. A methodological perspective has been chosen that uses strategies that allow us to know the phenomenon of addiction to gambling from the affected people themselves. Among the possible techniques, the Life Story and the In-Depth Interview have been chosen. Results. In general, we have observed that, sooner or later, the gambler is forced to confess the addiction to his family, since his gambling situation becomes untenable. In the same way, we have been able to see that, facing the problem must go beyond the laudable purpose of facing it individually, since this does not usually work, and that both family and professional help are necessary to be able to carry out the rehabilitation process with a certain guarantee of being able to overcome the addiction. Conclusions. It is easy to see that in order to stop gambling, a great change has to be made in the gambler's thinking, that is, to take seriously wanting to stop gambling and from there request the collaboration of professionals (including the Social Worker); help that must be complemented with family support, both being determined as the main factors to take into account in the recovery processes.

Keywords: Gambling addiction, Coping with the problem, Key Referents, Rehabilitation Process, Psychosocial Intervention.

\section{INTRODUCCIÓN}

Si bien desde el principio, tanto la APA (DSM III; IV y IV-TR) como la OMS (CIE-10 apartado F.63.0) otorgaron al juego patológico la consideración de trastorno del control de los impulsos, actualmente tras la revisión llevada a cabo por la APA del Manual de Diagnóstico y Estadístico de los Trastornos Mentales (DSM-5), éste queda contemplado dentro de una de las nuevas categorías denominada como "Trastornos relacionados a sustancias" incluyendo la patología del juego con el nominativo de "Trastorno por juego de apuestas". Aun contando con algunas voces disidentes con respecto a esta nueva nomenclatura (Cía, 2013; Choliz, 2014), no podemos menos que considerar esto como un hecho importante, dado el alcance que tiene dejar de ser valorado como un trastorno del control de los impulsos y pasar a ser reconocido como una enfermedad adictiva.

Tomando como referencia el DSM-5 (APA, 2013) vemos que la adicción al juego queda definida teniendo en cuenta, además de la pérdida del control ante el juego, la capacidad de generar problemas serios en todas las esferas de la vida del/a adicto/a. Ser adicto/a al juego tiene muchos costes (Chóliz y Lamas, 2017 (López y Estévez, 2019; Vázquez y Barrera, 2020; Torres Rodríguez, 2020; Álvarez Pérez, 2020; Rodríguez y García, 2021) ya que no sólo se van a ver resentidas las áreas personal y económica. La familia, el trabajo, la educación, las amistades y relaciones interpersonales y su propia salud física y 
psíquica, también van a sufrir las consecuencias que la adicción puede tener en ellas y así queda recogido entre los criterios diagnósticos a tener en cuenta a la hora de analizar esta enfermedad.

Aunque en las últimas décadas hemos asistido a una proliferación de todo lo que tiene que ver con el mundo que rodea al juego de apuestas (nuevos tipos), el verdadero motor de cambio ha venido propulsado por el juego online. La política liberalizadora llevada a cabo en las últimas décadas en Europa, ha ayudado a establecer una mayor permisividad y regulación de este sector, sobre todo en su variante online. De hecho en España, la aprobación de la nueva Ley de Regulación del Juego (Ley 13/2011, de 27 de mayo) trajo consigo una serie de novedades, que nos permiten hablar de una nueva configuración del juego en nuestro país. Cambios, que como bien señalan López y Estévez (2019), tienen que ver, sobre todo, con el incremento del número de operadores, de la oferta, de la publicidad y por ende del número de jugadores y/o jugadoras.

Actualmente se es consciente que la incursión de las TIC, sobre todo Internet, en nuestra vida cotidiana, no sólo ha tenido consecuencias muy positivas, sino que un uso mal adaptativo, ha conllevado para algunas personas efectos muy nocivos, dada la inferencia generadora y potenciadora que Internet ha tenido para el mundo de las adicciones conductuales, sobre todo en lo que respecta a la adicción a Internet, la adicción al juego en su modalidad online (Uchuypoma, 2017), la adicción a los videojuegos (Marco y Chóliz, 2014; Carbonell, 2020) y la adicción al cibersexo (Ballester et al, 2010).

Esta situación de incremento del juego online, sobre todo entre la población más joven (Chóliz y Lamas, 2017 ), nos obliga a tener que valorar, no sólo la magnitud (incidencia epidemiológica), sino también las consecuencias que lleva implícita esta problemática, así como, los procesos de tratamiento y/o rehabilitación que existen para dar solución a esta adicción. Actualmente tenemos a nuestro alcance toda una serie de instrumentos de diagnosis dirigidos, tanto a la población general, como de forma específica a los jóvenes y adolescentes (Entre otros: IGD-20, IGDS9-SF, POGQ, POGQ-SF, VAT, CVAT2.0, IGDS) (Bernaldo et al, 2020). No obstante se hace necesario apuntar que, aunque se tratan de instrumentos muy útiles para efectuar una primera aproximación genérica a poblaciones de riesgo, resultarían insuficientes para llevar a cabo un análisis pormenorizado de la problemática concreta de cada sujeto jugador o jugadora

Esto nos lleva a deducir que la evaluación individual de cada sujeto debe ser la premisa indispensable para llevar a cabo un buen diagnóstico; valoración que según explicitan Ibáñez y Saiz (2001), entre otros elementos deben contemplarse:

- El análisis riguroso de las consecuencias del juego, ya que la adicción al juego conlleva efectos en todos los ámbitos de la vida del adicto o adicta, tales como: el ámbito familiar, laboral, social económico, así como las posibles implicaciones legales que pudieran derivarse de la adicción. 
- La evaluación de la motivación del individuo cuando acude en busca de ayuda, dado que de ella dependerá gran parte del éxito del proceso de rehabilitación.

- El proceso de evaluación tiene por sí mismo implicaciones terapéuticas de gran importancia, ya que constituyen el primer contacto con el sujeto, de ahí que las primeras entrevistas resulten vitales, ya que cuando se establece una relación empática entre el profesional y el enfermo, se favorece el proceso terapéutico.

Es un hecho dado que, dentro de los programas de tratamiento o rehabilitación, la perspectiva social de la adicción es una dimensión que forma parte del proceso de evaluación, puesto que el juego patológico es una adicción generada en las estructuras sociales (Sánchez y Váldez, 2019). Tanto es así que el diagnóstico social se concibe como la conclusión elaborada teniendo en cuenta todas las pesquisas relevantes del usuario (social, familiar, laboral, personal, etc.). Información que nos va a permitir determinar de forma conjunta con el jugador o jugadora una serie de objetivos elaborados a partir de sus necesidades y así poder determinar el futuro tratamiento. Dentro de este proceso de intervención es necesario que los profesionales que se dedican a la elaboración del diagnóstico social (trabajadores sociales) tengan muy claro, cuáles deben ser las actitudes y aptitudes que debe mostrar para alcanzar un resultado objetivo y veraz de la situación de cada sujeto estudiado, como la necesidad de que la recogida de los datos sea exacta y muy minuciosa, teniendo para ello una serie de instrumentos básicos como son: la Ficha Social, el Informe Social, la Hoja de Seguimiento y la Ficha Laboral.

Diagnosticada la adicción, el nuevo interrogante que se nos plantea es ¿qué se puede hacer para abordar este problema tan complicado? Realmente ¿se puede dejar de jugar? La práctica profesional demuestra que sí. Dejar de jugar se puede hacer aunque no es tarea fácil. Para que esto sea factible es necesario contar con dos elementos claves: la familia y la ayuda profesional. Ambos elementos deben ser tenidos (sobre todo la familia) como centros estratégicos para el tratamiento de las adicciones. Tanto es así que, se ha evidenciado la importancia que tiene la colaboración e implicación de las familias para lograr, tanto el abandono de las conductas adictivas, como para llevar a cabo el seguimiento del tratamiento prescrito o la normalización del comportamiento (Caro y Plaza, 2016).

Importancia que también es recogida por la perspectiva antropológica de las adicciones. Para el Movimiento Rehumanizador, la familia ocupa un lugar privilegiado que necesita la sociedad para su regeneración, no sólo porque la rehumanización es ya de por sí preventiva, sino porque, como muy bien apunta Cañas $(2004,2013)$ en el seno de la familia es donde se puede curar mejor las heridas cuando ha fallado la prevención. Es necesario involucrar a la familia de forma paralela en todo programa terapéutico rehumanizador, lo cual implica ayudar a ésta a ser protagonista activa y no sociedad pasiva que se limite a asumir los cambios o a mostrarse recelosa con respecto del trabajo realizado por los demás.

A nivel educativo, la familia puede desempeñar un papel fundamental al erigirse como puente que une los programas terapéuticos educativos con el contexto general. Importancia que hay que extrapolar al 
ámbito socio-comunitario dado que el movimiento asociativo creado alrededor de los problemas adictivos funciona gracias al esfuerzo y la labor que desempeñan de forma desinteresada las familias. De ahí que actualmente, la red asociacionista se muestra como el mejor recurso que trabaja, la prevención, la recuperación y la rehabilitación, tanto de personas con problemas de adicción como de sus familias. De hecho se cree que, gran parte del éxito que tienen los grupos de autoayuda en el mantenimiento de la abstinencia reside en el efecto que éstos tienen sobre la construcción del YO del jugador y del Yo del familiar (Jaén y Garrido, 2004).

Pese a las posibles dificultades que algunas veces pueden darse en lo que tiene que ver con la articulación de los recursos profesionales en un contexto de asociacionismo de autoayuda, eso no quita para que las dos partes implicadas tengan muy claro su simbiosis. Dados los buenos resultados que están teniendo, algunos organismos públicos con competencias en esta materia, se han dado cuenta del potencial que adquiere el movimiento asociativo para trabajar la problemática de la adicción al juego. Cumplidos unos estándares de calidad, algunas asociaciones se han convertido en Centros Ambulatorios de Tratamiento del Juego Patológico, sirva como ejemplo la Asociación Onubense de Jugadores de Azar en Rehabilitación (AONUJER) de Huelva que desde 2009 desempeña su labor bajo la titularidad de Centro de Tratamiento Ambulatorio exclusivo de Juego Patológico, tras recibir la acreditación de la Consejería para la Igualdad y Bienestar Social.

\section{METODOLOGÍA}

Siendo consecuentes con las ventajas e inconvenientes en las que se mueve la investigación social, teniendo en cuanta el eje dicotómico cuantitativo/cualitativo, hemos optado por utilizar la perspectiva cualitativa, ya que ésta se mostraba mucho más eficaz para la consecución de nuestros intereses de estudio, ya que puede ofrecernos un contexto más amplio desde donde poder abordar un fenómeno tan complejo como es el de las adicciones sociales.

Concretamente elegimos la vertiente comprensivo-interpretativa, ya que ésta está centrada en analizar y comprender los hechos sociales desde el punto de vista de los propios afectados. De entre las diferentes técnicas elegimos la historia de vida y la entrevista en profundidad. Dado que el ámbito de estudio era la población afectada por la adicción al juego en proceso de rehabilitación (ludópata y familiar) que estuvieran vinculados a la Asociación Onubense de Jugadores de Azar en Rehabilitación (AONUJER), se llevaron a cabo 42 historias de vida: 22 a adictos/as y 20 a familiares. Teniendo en cuenta los familiares, la muestra conseguida fue de 16 para cónyuges/pareja, 3 para hijos y 1 para madre. Tras valorar el perfil de los usuarios se adoptaron una serie de criterios de selección que tuvieran en cuenta variables tan importantes como el sexo, la edad, la procedencia geográfica y el nivel de tratamiento en el que se encontraban en ese momento. Del mismo modo, para elegir a los familiares se estimó conveniente 
(si así era posible, ya que no todos obtienen el apoyo de éstos) elegir al familiar del jugador/a que previamente hubiera sido seleccionado en la muestra de jugadores/as.

Por su parte las entrevistas han sido realizadas, por un lado, al personal técnico que trabaja en la Asociación (Psicóloga y Trabajadora Social), y por otra, al personal que dirige la asociación (un total de 8 entrevistas). Resaltar que éstos últimos fueron una gran fuente de información, ya que pudimos obtener datos, teniendo en cuenta, no sólo, los roles que éstos enmarcan dentro del ámbito organizacional: exjugador/familiar, todos ellos pioneros que iniciaron y lucharon por conseguir una respuesta factible a su problema de juego, sino también, por su papel como monitores de acogida y terapia, que bajo el rol de coterapeutas complementan la labor que llevan a cabo los profesionales que trabajan dentro de la entidad.

\section{RESULTADOS}

\subsection{AFRONTANDO EL PROBLEMA}

¿Es posible dejar de jugar? ¿Existe una salida real para este problema? Si tenemos en cuenta que a nada le precede la finitud, sino todo lo contrario, que llegado el tiempo las situaciones tienden a cambiar, aunque este cambio no obre por sí mismo, la respuesta a los anteriores interrogantes es Sí. De manera general, la decisión de dejar de jugar viene precedida por algún elemento o circunstancia que hace que la adicción al juego termine haciéndose evidente. De hecho la información obtenida hace evidente que toda situación límite lleva implícita un proceso de desesperación donde el adicto o adicta acaba superado y acorralado por las circunstancias que le obligan a tener que decidir qué hacer. Tanto es así que, a partir de ese momento queda supeditada a una doble decisión: confesar el problema (si la familia aún no lo sabe) y pedir ayuda, o huir de la situación, intentando acabar con ésta de modo dramático.

\footnotetext{
"Mi período de juego fue mentira sobre mentira, destruyendo la economía familiar. Problemas de tipo judicial. Deudas con el banco llegó un momento en que no me pagaban la nómina. Yo había dejado de pagar préstamos, tenía mi piso embargado, tenía la nómina embargada, y al final no me quedo otra que confesarlo todo (Jugador, 55 años).

"Me encontraba tan mal que una vez me tomé pastillas. Sólo una vez lo intenté y me ha pesado mucho. El no pensar en mis hijos cuando lo intenté me ha dolido mucho y lo estoy superando desde que llegué aquí. Ellos me están ayudando. Yo quiero curarme y hago todo lo que ellos me digan" (Jugadora, 39 años).
}

Evidenciado el problema la cuestión siguiente pasaría por ¿qué se puede hacer ante una situación de tal envergadura? La tendencia natural lleva a querer encarar el problema de forma personal. De hecho este es el argumento que el propio adicto o adicta va a utilizar de cara a su familia para convencerla de que, si él se lo propone, puede dejar de jugar sin requerir la ayuda de nadie.

"Es a lo primero que te agarras. Lo primero es creer que tú, si quieres, lo puedes dejar, pero es otra de las mentiras que te sacas de la manga. Aunque te lo propones y lo cumples durante unos días, al final acabas cayendo en lo mismo y más jodido todavía” (Jugador, 40 años). 
En principio, esta solución suele ser aceptada por la familia, ya que la mayoría desconoce la magnitud del tema que tienen entre-manos. De hecho todos los casos analizados, aludieron haber llevado a cabo esta fórmula para dejar de jugar, la cuestión es que a nadie le funcionó. La razón de este fracaso hay que situarla en el hecho de que la determinación de dejar el juego no llevaba el respaldo de una decisión propia, sino que nació desde la imposición, como contrapartida para seguir formando parte de la familia.

\begin{abstract}
"Yo intenté quitarme solo, dos o tres veces y estuve un mes y medio, incluso dos sin entrar en ningún salón, pero cuando ya no podía más entraba. Yo me he ido llorando del salón. Yo salía de alli fatal. Muchas veces decía: "yo me quito de esto por cojones porque esto no puedo conmigo" y entonces vine ya, pero yo no quería venir. Vine obligao. Yo no quería venir, gritando que no quería venir, pero ya me vi como un desgraciao porque mi mujer me quiso dejar y... vine por probar" (Jugador, 34 años).
\end{abstract}

Salvado este obstáculo, todos son conscientes del giro que a partir de ese momento va a tomar sus vidas: dejar de jugar no es fácil pero sí es una tarea factible. Consecuentemente esto lleva implícito la obligación de responsabilizarse de la determinación tomada. Pero esta decisión no afecta sólo al jugador o jugadora, la familia como -víctima colateral- también debe de ponerse en tratamiento.

\begin{abstract}
"Yo entendí que el único responsable de mi rehabilitación era yo. Yo tuve que ser consciente de eso y así se lo hice saber a mi familia. Ella (familia) también me ayudó mucho y desde el primer día estuvieron apoyándome. Desde el principio mi mujer vino a la asociación y hemos seguido todos los pasos que la psicóloga nos ha ido diciendo. Ella le dijo a mi mujer que tenía que comprometerse si quería que yo saliera del problema y ella desde siempre ha estado a mi lado" (Jugador, 38 años).
\end{abstract}

Afrontar el problema es muy difícil. Tanto la familia como el adicto o adicta sabe que esto es una tarea encomiable que requiere: voluntad de poder y tiempo. Voluntad para deshacer una vida basada en el juego y tiempo para edificar una nueva vida basada en el no juego. Pero, para encauzar este nuevo proyecto necesitan "hacer suyas" algunas cuestiones básicas, prescritas por los terapeutas, sobre las que se asienta cualquier proceso de tratamiento. Ejemplo de ello tenemos el pensamiento o la idea de creer que su problema tiene solución, pero para ello es necesario acabar con la causa primaria que originó el problema de la adicción. Como hemos podido comprobar en la primera parte de este análisis, en la mayoría de los casos la ludopatía nace al amparo de otras dificultades. Problemas que deben ser resueltos si verdaderamente se quiere dejar de jugar. Todos saben que tomar conciencia del porqué se empezó a jugar es vital para emprender el camino de la rehabilitación.

"El proceso de tratamiento es complicado mucho más de lo que creen en un principio. En la primera entrevista, conmigo, se le deja claro todo lo que conlleva el proceso de rehabilitación. De todo ello se habla, tanto con el afectado, como con la familia. A partir de ahí todo depende de ellos. Es importante que todos estén concienciados de la necesidad de apoyar a los profesionales en su proceso de rehabilitación" (Psicóloga AONUJER). 


\subsection{PRINCIPIOS QUE REGULAN EL PROCESO DE REHABILITACIÓN}

Tomada la decisión e iniciado el camino hacia el cambio, es importante que los sujetos implicados sepan con claridad cuáles van a ser las nuevas reglas del juego. Tratar la adición no implica sólo predisposición o condescendencia; se requiere de otros elementos como el apoyo y la disciplina. Tomarse en serio la idea y contar con apoyos suelen ser las claves que determinan el éxito o el fracaso en el proceso de tratamiento. Encarar esta nueva etapa es duro y mucho más si se la tienen que llevar en soledad, de ahí la gran importancia que adquiere la familia en todo el proceso de rehabilitación. Tanto el varón como la mujer ludópata son conscientes de la importancia que tiene la familia en su proceso de rehabilitación. Haber intentado dejar de jugar sin la ayuda de un familiar y profesional, es algo muy común en ellos, pero poco efectivo, de ahí que cuando tienen claro dejar de jugar lo hagan solicitando el apoyo de ambos.

\footnotetext{
"Yo lo tengo muy claro, muy claro. Si mi mujer y mis hijos no hubiesen estado ahí, yo ahora no sé qué hubiese sido de mí. No sé dónde estaría en estos momentos, bueno sí jugando como siempre" (Jugador, 40 años).
}

No obstante la realidad nos ha mostrado que no todos cuentan con esta ayuda. La mujer adicta al juego es la que más va a sufrir la falta de apoyo familiar. Tanto por boca propia, como de la de los profesionales, se nos ha hecho partícipes del apunte de lo que lo normal pasa por ver a la mujer enfrentándose a su adición en la más estricta soledad o con la ayuda de alguna amistad.

\footnotetext{
"Mi marido no me ha ayudado en nada, en nada. Él vino aquí el primer día y el segundo le dijo a la mujer de Jorge (monitora) que esto lo veía una tontería. En nada, en nada. Yo lo he llevado todo sola. Ni siquiera me preguntaba cómo me iba” (Jugadora, 39 años).
}

En otros casos el apoyo puede venir de la mano de los hijos. AL margen de lo vivido hasta este momento, los hijos tienden a olvidar y perdonar más fácilmente todo lo pasado que la propia pareja. De ahí que sin pensarlo apoyen cualquier decisión que vaya encaminada a dejar la adicción.

Mi hija me buscó donde ir a curarme porque la cosa ya era imposible (...) En verdad con mis hijos no es que estuviesen las relaciones cortadas pero no había fluidez y sin embargo siempre han estado ahí ayudándome. El que falló fui yo. (Jugador, 66 años).

Por último no podemos dejar de plantear ¿qué pasa cuando el afectado por la adición es un hijo? La realidad estudiada nos ha mostrado que la responsabilidad del problema recae principalmente en la figura de la madre. Aunque el padre suele tomarse muy mal el problema del hijo/a, éste tiende a delegar toda responsabilidad en la madre, pues suele tener claro que es a ella a quien le corresponde el cuidado de sus hijos e hijas. 
verdad es que me vi muy sola sin saber que hacer, muerta de miedo, porque yo no sabía ni que era eso del juego". (Familiar, 58 años).

\section{DISCUSIÓN Y CONCLUSIONES}

Los resultados extraídos del anterior análisis, nos llevan a concluir que tarde o temprano la persona adicta al juego se ve obligada a tener que confesar el problema, movida fundamentalmente por que haya sido descubierta por un familiar, porque ha intentado suicidarse o porque llevado, por un momento de lucidez, ha sido capaz de pedir ayuda. De hecho aunque parezca sencillo, hemos podido evidenciar que no lo es. No lo es, porque aunque todo parezca estar a favor de iniciar el proceso de cambio, en el fondo son muy pocos los que desean que eso sea así. Sin bien prometen dejar de jugar (una vez han sido conscientes de la dificultad de hacerlo ellos solos) accediendo a la petición familiar de ponerse en manos de profesionales, solo es apariencia. Imagen que les va a permitir bajar la tensión por un tiempo, mientras planean seguir jugando.

En cuanto a los motivos que facilitan el proceso de cambio vemos que éstos lo sitúan en el propio carácter que toma el proceso de ayuda, ya que secundados o apoyados por la familia y por los profesionales van a ser capaces de avistar una solución eficaz a su problema de adicción.

Al hilo de las anteriores conclusiones y teniendo en cuenta la literatura existente sobre este tema, podemos ver, que la recuperación natural puede ser un hecho factible. De hecho, Carballo et al (2004) manifiestan que la recuperación natural en este tipo de problemas, es un fenómeno más habitual de lo que en un principio se pudiera creer. Hodgins y El-Guebaly (2000) llevaron a cabo, durante un año, un estudio con jugadores patológicos cuyos resultados mostraron que el $80 \%$ había conseguido bajar de forma significativa su juego y el $25 \%$ consiguió llegar a la abstemia. De igual modo, estos autores mostraron que esta fórmula es mucho más eficaz en situaciones donde la problemática del juego no era muy grave.

Otro estudio que mostró que la recuperación natural no es una excepción, es el realizado por Slutske, Jackson y Sher (2003). En un período de 11 años, estos autores llevaron a cabo cuatro evaluaciones a adolescentes que pasaron a ser adultos y que tenían problemas de juego. Los resultados mostraron que sólo entre un 2\% y un 5\% mantuvieron sus problemas de juego. El juego llegó a significar más un trastorno episódico transitorio que crónico y estable.

Asimismo, el estudio longitudinal efectuado por De Fuentes-Merillas et al (2004), cuyo objetivo fue evaluar la estabilidad temporal a lo largo de dos años de jugadores patológicos, concluyó con una tasa estimada (a partir de cifras de estabilidad de la adicción) de que entre el 57,1\% y el 89,9\% redujo su conducta de juego, y en muchos de ellos la recuperación tuvo lugar sin que mediara tipo alguno de tratamiento profesional.

Por su parte, la investigación llevada a cabo por Shaffer y Hall (2002) para evaluar los problemas de juego y alcohol que tenían una muestra de empelados de casinos, concluyó con que el 22,6\% de los 
sujetos había reducido de forma sustancial su conducta de juego y el consumo de alcohol de forma natural. Además este trabajo mostró la existencia de un proceso a través del cual los sujetos podían pasar de unas categorías de severidad a otras (previamente los autores distinguieron tres niveles de dureza en función de la gravedad del problema) teniendo en cuenta la doble modalidad: de niveles más graves a patrones de juego de menor severidad y al contrario.

Es difícil comparar los niveles de recuperación operados entre ambas opciones. En parte, estas dificultades se deben, como bien apuntan Toneatto y Ladouceur (2003), a la falta de conocimientos fiables de los mismos, dado que muchos de los estudios realizados han carecido de importantes limitaciones metodológicas (muestras demasiado pequeñas o el empleo de programas poco estructurados). Tampoco se ha estudiado con ahínco, si el resultado de la recuperación natural es estable o si se aperciben diferencias entre sus tasas de recaída. En relación a esto último, Nathan (2003) y Timko et al (2000), pusieron de manifiesto que los jugadores que se recuperan sin tratamiento formal tienen mayores posibilidades de recaer que los que lo han hecho con ayuda profesional.

De igual modo se ha podido comprobar que, por sí mismas, las intervenciones autodirigidas (grupos de jugadores) tampoco suelen ser eficaces, aunque suelen ser un buen complemento a la terapia psicológica (Álvarez Pérez, 2020). De hecho hemos podido comprobar que la mayoría de los tratamientos, ya sean ambulatorios o en régimen de internamiento, están orientados hacia el consejo profesional, las psicoterapias grupales y la asistencia a grupos de autoayuda como "jugadores en rehabilitción" (Blanco, 2014)

Aunque también resultaría de interés conocer el perfil de los jugadores que acuden y los que no acuden a tratamiento, al día de hoy tampoco existen muchos datos sobre este tema. Entre las posibles variables se barajan la "severidad del problema" (a mayor severidad más probabilidad de recurrir a un tratamiento de recuperación profesional) (Hodgins y El-Guebaly, 2000); una mayor frecuencia de “trastornos mentales" y "abuso de sustancias" en comparación con los sujetos que recurrían a la ayuda formal (Marotta, 1999): así como un nivel mayor de "trastornos afectivos" como la depresión (el 75\% de los jugadores que acudía a tratamiento sufría de depresión) (Blaszczynski y Silove, 1995), o el estrés psicológico causado por los propios problemas del juego (Marotta, 1999).

Prácticamente, la atención en este tema (recuperación natural) se ha centrado en valorar los procesos de cambio y mantenimiento de los logros obtenidos a largo plazo. Ambas cuestiones muy importantes para ayudar a la mejora de los tratamientos de la adicción al juego. Destacar que, apenas sí se han encontrado diferencias entre los jugadores que se recuperan por sí solos y los que acuden a tratamiento. Como estrategias de auto-cambio, Carballo et al (2004), señalan a todas aquellas que tienen que ver con los problemas emocionales (estrés, pánico, depresión, y culpa) y financieros (pérdidas provocadas por el juego). Por su parte, los eventos vitales no parecen jugar un papel importante como 
precipitante del cambio, sino que éstos aparecen una vez recuperado el jugador, ayudándoles a mantener la abstinencia.

Dentro de las estrategias o factores que avalan el mantenimiento del cambio, Hodgins y ElGuebaly (2000), apuntan sobre todo a las habilidades conductuales y a las cognitivo-motivacionales. Entre las primeras resaltan el control de los impulsos (evitación de los lugares que implican situación de riesgo) y la realización de actividades no relacionadas con el juego (ejercicio, lectura, estar con la familia e implicarse más en el trabajo). Entre las segundas se recogen principalmente: recordar los problemas que les causó el juego en el pasado, anticipar los problemas que les volvería volver a jugar y el uso de la fuerza de voluntad.

En referencia a los procesos de cambio, Hodgins (2001), mostró que los más usados por jugadores patológicos recuperados por sí mismos, fueron los de tipo cognitivo, en concreto la autorreevaluación (sentirse alterado cuando pensaba en sus problemas de juego, sentirse avergonzado de las cosas que hacía cuando jugaba y resistirse a la auto-imagen de jugador), reevaluación ambiental (reconocimiento del impacto negativo del juego sobre otras personas), el relieve dramático (experimentación de fuertes sentimientos negativos sobre la implicación personal con el juego) y la auto-liberación (proceso de compromiso con uno mismo para la acción y el recordatorio de que el éxito es posible); mientras que los menos utilizados fueron el manejo de contingencias y la liberación social. Mientras que el grupo de recuperados con ayuda profesional, referían mucho más un aumento de la concienciación, la autorreevaluación, la liberación social, las relaciones de ayuda, el control de los estímulos, la reevaluación ambiental y el relieve dramático.

Otro de los factores de mantenimiento más adoptado, por el grupo de recuperación natural, es el apoyo social por parte de la familia y los amigos. En un estudio llevado a cabo por Hodgins y El-Guebaly (2000), destacaron que el 30\% de los casos de recuperación sin tratamiento establecieron el apoyo como un factor definitivo en su proceso de mantenimiento hacia el cambio. De hecho, son muchas las terapias que centran su actuación en el contexto familiar dada que la implicación de ésta para lograr, no sólo el abandono de las conductas adictivas, sino también para el seguimiento del tratamiento prescrito y la normalización del comportamiento, es vital (Caro et al, 2016).

Por último apuntar que superar la adicción al juego resulta una tarea bastante ardua, pero no imposible y esto es lo que debe de quedar en la mente de cualquier persona adicta o familiar vencida por la adicción al juego. Dejar de jugar es posible, tan solo tiene que tener determinación y apoyos (familiar/profesional). Dominar la adicción implica enfrentarse a ella, hacer visibles los miedos que un día llevaron a tomar este camino equivocado y darse un voto de confianza para poder salir adelante. Y en este salir adelante tener claro que tanto la familia como los profesionales, van a quedar determinados como los factores claves en el proceso de recuperación o rehabilitación. 


\section{BIBLIOGRAFIA}

Álvarez Pérez, A. (2020). Revisión del Juego Patológico: Modelos, déficits y tratamiento. Revista Española de Drogodependencias, (45 (3), 8-26.

APA (2013). Diagnostic and Statistical Manual of Mental Disorders (DSM-V). (Fifth Edition). Washington, DC: American Psychiatric Association.

Ballester Arnal, B.; Gil Llario, M.D.; Gómez Martínez S. \& Gil Juliá, B. (2010). Propiedades Psicométricas de un instrumento de evaluación de la adicción al cibersexo. Psicothema, 22 (4), 1048-1053. ISSN: 0214-9915

Bernaldo De Quirós, M., Labrador Méndez, L. Sánchez Iglesias, I. \& Labrador, F.J. (2020). Measurement instruments of Internet gaming disorder in adolescents and Young people according to DSM-5 criteria: a systematic review. Adicciones, 32 (4), 291-302.

Blanco Miguel, P. (2014). El Estudio de las Adicciones Sociales. El juego como Adicción Social: problemática Social, Contexto Familiar y Movimiento Asociativo. El caso de Huelva. Tesis Inédita Universidad de Huelva. Huelva. España.

Blaszczynski, A \&Silove, D. (1995). Coghitive and behavioral therapies of pathological gambling. Journal of Gambling Studies, 11, 195-219.

Cañas Fernández, J.L. (2004). Antropología de las Adicciones. Psicoterapia y Rehumanización. Madrid: Dykinson.

Cañas, Fernández, J.L. (2013). Psicoterapia y Rehumanización de las Adicciones. Un modelo para la Bioética Personalista. Cuadernos de Bioética, XXIV, 1ª , 101-112.

Carballo, J.L., Secades, R., Fernández, R., García, O. \& Sobell, L. (2004). Recuperación de los problemas de juego patológico con y sin tratamiento. Salud y Drogas, 4 (2), 61-78. ISSN: 1578-5319. ISSNe 1988-205X.

Carbonell, X. (2020). El Diagnóstico de la Adicción a videojuegos en el DSM-5 y la CIE-11: Retos y oportunidades para clínicos. Papeles del Psicólogo. Pychologist Papers, 41 (3), 211-218. http://doi.org/10.23923/pap.psicol2020.2935.

Caro Amada, C. \& Plaza de la Hoz, J. (2016). Intervención Educativa Familiar y Terapia Sistémica en la Adicción Adolescente a Internet: Fundamentación Teórica. REOP, 27 (1), 99-113. ISNN electrónico: 19897448.

Chóliz, M. (2014). Historia de una adicción: del vicio al trastorno del juego. C. Med. Psicosom, $N^{o} 11$, 84-88.

Chóliz, M. \& Lamas, J. (2017). “¡Hagan Juego, menores!” Frecuencia del juego en menores de edad y su relación con indicadores de adicción al juego. Revista Española de Drogodependencias, 42 (1), 34-47.

Cía, A.H. (2013). Las Adicciones no relacionadas a sustancias (DSM-5, APA, 2013): Un primer paso hacia la inclusión de las Adicciones Conductuales en las clasificaciones categoriales vigentes. Revista de Neuropsiquiatría 76 (4), 210-217.

De Fuentes-Merilla, L., Koeter, M. W., Schippers, G.M. \& Van den Brink, W. (2004). Tmporal stability of pathological scratchcard gambling among adul scrathcard two years later. Adiccition, 99, 117-127.

Hodgins, D.C. \& El-Guebaly, N. (2000). Natural and treatment-assisted recovery from grambling problems: A comparison of resolved and active gamblers. Addiction, 95, 777-789. 
Ibañez, A. \& Saiz-Ruiz, J. (2001). La Ludopatía una "nueva enfermedad”. Barcelona: Masson.

Jaén Rincón P. \& Garrido Fernández, M. (2004). "El juego en la pareja: desarrollo de un modelo explicativo de los patrones de construcción deconstrucción y reconstrucción del vínculo matrimonial".

En: Garrido Fernández, M., Jaén Rincón, P. \& Domínguez, Álvarez, A. (Comps) (2004). Ludopatía y Relaciones Familiares. Barcelona: Paidós.

López-González, H. \& Estévez, A. (2019). ¿Está aumentando la población adicta al juego?. Norte de Salud Mental, vol. XVI, nº 60, 77-83.

Marco, C. \& Chóliz, M. (2014). Tratamiento cognitivo conductual de la adicción a videojuegos de rol online: Fundamentos de propuesta de tratamiento y estudio de caso. Anales de Psicología, 30 (1)46-55. DOI: http:// dx.doi.org/10.6018/analesps30.1.150851.

Marotta, J.J. (1999) Recovery from gambling problems with and without treatment (natural resolution). Dissertations Abstracts Inernational: Sección B: The Sciences \& Engineering, 60, 29-52.

Nathan, P.E. (2003). The Role of Natural Recovery in Alcoholismand Pathological Gambling. Journal of Gambling Studies, 19, 279-286.

Rodríguez Rodríguez, M. \& García Padilla, F.M. (2021). El uso del videojuego en adolescentes. Un Problema de Salud Pública. Enfermería Global, 62, 557-574. Doi: https://doi.org/10.6018/eglobal.438641.

Sánchez Ramos, R. \& Váldez Montero, C. (2019). La problemática social del juego patológico: un nuevo campo de exploración para el Trabajo Social. Margen. Revista de Trabajo Social y Ciencias Sociales, 94, 18.

Shaffer, H.J. \& Hall, M.N. (2002). The natural history of gambling and drinking problems among casino employees. Journal os Social Psychology, 142, 405-424.

Slutske, WS., Jackson, K.M. \& Sher, K.J. (2003). The natural history of gambling and drinking problems among casino employees. Journal of Social Pychology, 142, 405-424.

Uchuypoma Canchumani, D. (2017). Juegos online: Una mirada desde 1 ljuego patológico. Hamut `ay, 4 (2), 55-64. Recuperado de: http://dx.doi.org/10.21503/hamu.v4i2.1472.

Vázquez-Fernández, MJ. \& Barrera-Algarín E. (2020). El Juego online en España y las apuestas deportivas: Los jóvenes como nuevos perfiles con ludopatía. Health and Addictions. Salud y Drogas, 20 (2), 61-69. ISSN: 1578-5319. ISSNe 1988-205X.

Timko, C., Moos, R.H., Finney, J.W. \& Lesar, M.D. (2000). Long-term outcomes of alcohol use disrders: Comparing untreated individuals with those in alcoholics anonymus and formal treatment. Journal of Studies on Alcohol, 61, 529-540.

Toneatto, T. \& Ladoceur, R. (2003). Treatment of Pathological Gambling: A Critical Review of the Literature. Psychology of Adidictive Behaviors, 17, 284-292.

Torres-Rodríguez, A. (2020). Cambios Psicológicos en una muestra clínica de adolescentes con Trastorno de Juego por Internet. Revista Española de Drogodependencias, 45 (4), 29-48. 